\title{
श्रीमद्भागवत में योग के विविध आयाम
}

रश्मि शर्मा

सारांश

योग ब्रह्मा द्वारा निर्दिष्ट एक शाश्वत विज्ञान है, साधना पद्धति है। जो मनुष्य को सभी प्रकार के आवरणों एवं विक्षेपों से सदा के लिये मुक्त करता हुआ ऐसा विशुद्ध अंतःकरण वाला बना देता है कि परमात्मा से उसका अभिन्न सम्बन्ध स्वतः ही स्थापित हो जाता है। पौराणिक साहित्य जनसाधारण के समक्ष बहुत ही सहज ढ़ग से ऋषियों द्वारा प्रतिपादित योग के इसी उद्देश्य को प्रस्तुत करता है। श्रीमद्भागवत महापुराण जहाँ एक ओर कथाओं के माध्यम से जनसाधारण को परमात्मा की भक्ति की ओर आकर्षित करता है, वहीं दूसरी ओर योग के गूढ़तम रहस्यों का प्रतिपादन करते हुए उसके विविध आयामों का विवेचन करता है। भागवत में ज्ञानयोग, भक्तियोग, कर्मयोग व अष्टांगयोग के माध्यम से कृष्ण तत्व का ही वर्णन प्राप्त होता है। श्रीमद्भागवत ज्ञानयोग के अन्तर्गत समस्त वृत्तियों से परे निर्गुण ब्रह्म तत्व का विवेचन हुआ है। श्रीमद्भागवत की एक विशेषता यह है कि इसमें भक्तिसंगत ज्ञान का वर्णन है। कर्मयोग के अन्तर्गत कर्म को फलभोग का हेतु माना गया है। यद्यपि कर्ता भगवान श्रीकृष्ण ही हैं परन्तु मायारूपी अविद्या के कारण ही जीवों को कर्तापन की भ्रांति होती है। इसीलिए इसमें अपने समस्त कर्मों को भक्ति भाव से भगवान श्रीकष्ण में समर्पित करने की बात कही गयी है। श्रीमद्भागवत में वर्णित अष्टांगयोग उपनिषदों और पातंजल योगसूत्र के अष्टांगयोग का प्रतिनिधित्व करता है। इसमें वर्णित अष्टांगयोग में भी भक्ति का सम्पुट है। इस तरह यह राजयोग और भक्तियोग का अनूठा समन्वय प्रस्तुत करता है। प्रस्तुत शोधपत्र का उद्देश्य जनसाधारण को श्रीमद्भागवत महापुराण में वर्णित योग की विविध धाराओं से अवगत कराना तथा इस सहज मार्ग की ओर जीवन की दिशा धारा को प्रेरित कराना है।

कूट शब्द : पौराणिक साहित्य, योग के विविध आयाम, ज्ञानयोग, कर्मयोग, भक्तियोग एवं अष्टांगयोग।

मनुष्य जीवन इस संसार का सर्वोत्कृष्ट वरदान है; क्योंकि मनुष्य योनि में ही अधिकतम विकास की पूर्ण संभावनाएँ हैं। मनुष्य की बहुमूल्यता का अनुभव हो जाए, तो जीवन में आने वाली सारी कठिनाइयाँ, कष्ट, विघ्न-बाधायें, हमें हमारे उद्देश्य से भ्रमित नहीं कर सकती हैं। जीवन की सार्थकता उसके अपने दृष्टिकोण में है, साथ ही उसके द्वारा भावप्रवणता से किए गए कर्म में है। जीवन का आनंद किसी वस्तु या परिस्थिति में नहीं, बल्कि जीने वाले के दृष्टिकोण में हैं। जब हमारा दृष्टिकोण परिवर्तित हो जाता है, तब हमारा जीवन आनंदमय हो जाता है और फिर सत्कर्मों के माध्यम से हमारे चित्त पर छाए कुसंस्कार हटते चले जाते हैं और इस प्रक्रिया से चित्त शुद्धि के सिद्धि के आयाम प्रकट होते हैं और आत्मिक अभ्युदय के फलस्वरूप व्यक्ति, व्यक्तित्व के केंद्र से दूर जाने के स्थान पर अपने केंद्र की तरफ लौटता है, जहाँ उसे योग की अनुभूति होती है।

यद्यपि साधारण मनुष्य को जब यह मालूम होता है कि परमात्मा देश, काल और वस्तु-भेदों से परे, हमारी बुद्धि एवं इंद्रियों से अतीत, अपने स्वतः सिद्ध स्वरुप में स्थित है, तब वह यह सोचकर भयभीत हो जाता है कि जो हमारी वृत्तियों के आकलन से सर्वथा अतीत है, उसकी हम उपासना कैसे करें? उस परमतत्व से योग कैसे हो? मनुष्य कि इस विवशता को महर्षि व्यास ने भली-भांति अनुभव किया और उन्होंने भगवान की कृपालुता का आश्रय लेकर उनकी सर्वव्यापकता एवं सर्वात्मकता
के यथार्थ आधार पर देश, काल और वस्तुओं के भीतर ही भगवान के सानिध्य, उपासना और र्मृति का ऐसा प्रशस्त द्वार पौराणिक साहित्य के माध्यम से उद्धाटित किया, जिसे देखकर उनके सामने कृतज्ञता के भार से सिर स्वयं ही अवनत हो जाता है।

पौराणिक साहित्य भारत का सच्चा इतिहास है। इनसे ही भारतीय जीवन का आदर्श, भारत की सभ्यता, संस्कृति तथा भारत के विद्या वैभव के उत्कर्ष का वास्तविक ज्ञान प्राप्त हो सकता है। पुराण शब्द का अर्थ है- जो वृतान्त पहले हो गया हो, उसका जिसमें वर्णन हो वही पुराण है। वायु पुराण में कहा गया है-

यरमात् पुरा हृयनतीदं पुराणं तेन तत् सृतम्।

निरूक्तमस्य यो वेद सर्वपापे: प्रमुच्यते।/ (वायुपुराण, 1/203) अर्थात् प्राचीन काल से प्राणित होने के कारण यह पुराण कहा जाता है। जो इसकी व्याख्या जानता है, वह समस्त पापों से मुक्त हो जाता है।

वास्तव में वैदिक वांग्मय में वर्णित विषयों का रहस्य पुराणों में उपाख्यानों द्वारा सुस्पष्ट किया गया है। अतः वेदनिहित तत्वों की जानकारी के लिये पौराणिक साहित्य का श्रवण-मनन अत्यावश्यक है। पुराणों के परिज्ञान के बिना वेद, वेदांग, उपनिषद का ज्ञाता भी ज्ञानवान नहीं माना गया है (ब्रह्माण्ड प्रक्रिया, 1/170; वायुपुराण पूर्वार्द्ध, 1/45)। इसीलिये श्रीमद्भागवत में भी इतिहास पुराण को पंचम वेद कहा गया है। इतिहास पुराणं च 
पंचमो वेद उच्यते' (श्रीमद्भागवत, $1 / 4 / 20$ )। तात्पर्य यह है कि पुराण भी वेद के समान नित्य हैं।

\section{पौराणिक साहित्य में 'श्रीमदभागवत' का प्राकट्य और स्थान} आर्य जाति में सब प्रकार की उन्नति के लिए प्राय: दो प्रकार के शास्त्र स्वीकार किये गये हैं- श्रुति और स्मृति। श्रुति के शब्द नित्य होते हैं। सब युग मनवन्तर और कल्पों में उनकी आनुपूर्वी एक सी रहती है। सृष्टि के आरम्भ में प्रणव, गायत्री और मन्त्र संहिता के रूप में उनका अनाहत नाद होता है। विशुद्ध अन्तःकरण वाले ऋषिगण उसका श्रवण करते हैं और पीछे अपने शिष्य परम्परा में उन्हीं शब्दों में उनका विस्तार करते हैं। दूसरे प्रकार के शास्त्र 'स्मृति' कहलाते हैं। मन्वादि स्मृति, महाभारत आदि इतिहास, श्रीमद् भागवतादि महापुराण र्मृति शास्त्र के अन्तर्गत हैं। महान तपस्वी ऋषियों के परम पवित्र अन्तःकरण में भगवान की प्रेरणा से इन भावों का आविर्भाव हुआ करता है। ये शारत्र भाव रूप से सर्वदा एक ही रहते हैं परन्तु इनके शब्दों की आनुपूर्वी परिवर्तित होती रहती है। सृष्टि के प्रारम्भ में प्राचीन भावों की र्मृति होती है और र्मृति के आधार पर रचे जाने के कारण वे 'स्मृतिशास्त्र' कहलाते हैं (पोद्दार, 1916, पृ0 49)। श्रीमद्भागवत महापुराण ऐसा ही स्मृति शास्त्र है जिसका संस्कृत साहित्य और विषेशतः पुराण साहित्य में निराला स्थान है।

श्रीमद्भागवत भगवद्तत्व को प्रकाशित करने वाला ऐसा ही अतुलनीय दीपक है। भगवान नारायण ने सृष्टि के प्रारम्भ में ब्रह्मा को करूणावश इस ज्ञान प्रदीप का दान किया था (श्रीमद्भागवत, $12 / 13 / 19)$ । उन्होनें ही ब्रह्मा के रूप से नारद को, नारद के रूप से व्यास को, व्यास के रूप शुकदेव को और शुकदेव के रूप से राजा परीक्षित को यह ज्ञान दिया। ब्रह्मनदी सरस्वती के पश्चिम तट पर व्यास जी के शम्याप्रास नामक आश्रम में ब्रह्म विद्या श्रीमद्भागवत की रचना हुई (श्रीमद्भागवत, महात्मय, 1/6)। श्रीमद्भागवत में गहरी आध्यात्मिक रहस्यों के संकेत छीपे हुये हैं। इस ग्रन्थ में ज्ञान, कर्म और भक्ति का अद्भूत समन्वय है। यह वैदिक साहित्य और यौगिक ग्रन्थों के गहन विषयों का खुला रहस्य है, इसमें आध्यात्मिक, आधिदैविक और आधिभौतिक विज्ञान त्रय का बड़ा मनोरंजक वर्णन है। इसमें भारत के इतिहास के बहाने वैदों का रहस्य खोला गया है। भागवत के प्रत्येक अक्षर से एक अतीव सुन्दर अर्थ की उद्धरणा की जा सकती है। 'भा' अक्षर से भारूप प्रकाश स्वरूप सच्चिदानन्द का संकेत किया गया है- भा प्रकाशे चिदानन्दे, 'ग' अक्षर से उस भगवान के विषय में लौकिक गति का अर्थ निकलता है- गतिर्मस्यात्र लौकिकी, 'व' अक्षर भागवत को सब शास्त्रों में वरिष्ठ-श्रेष्ठ बतलाता है- वरिष्ठ सर्वशास्त्राणों, 'त' अन्तिम अक्षर इस बात की ओर संकेत कर रही है कि यह ग्रन्थ इस भवार्णव से पार जाने के लिए एक मात्र तरणि-नाव है- तरीणभूभूतभवार्षवे। (पोद्दार, 1910, पृ० 143)।
इस प्रकार भागवत के अक्षरों की इस भावमयी सांकेतिक व्याख्या का यही अर्थ है कि यह ग्रन्थ प्रकाशरूप परमात्मा के विषय में लौकिक उपाय को बतलाने वाला है, सब शास्त्रों से बढ़कर है तथा इस संसार रूपी समुद्र से पार जाने के लिए एक सुदृढ़ नौका का कार्य करता है। श्रीमद्भागवत महापुराण जिसे हम मात्र पठन-पाठन, श्रवण-कीर्तन व मनन-प्रवचन आदि का ही विषय मानते हैं, उसमें भी यौगिक प्रणालियों का इस प्रकार वर्णन मिलता है कि कोई भी सहज और सरल तरीके से इसे अपनाकर अंतरंग चेतना का परिष्कार करते हुए योग के परम लक्ष्य को प्राप्त कर सकता है। वर्तमान युग में श्रीमद्भागवत का श्रवण-मनन भी अति प्रासंगिक है क्योंकि इस की प्राप्ति सरल व सुगम है। यह साधना एक प्रकार से मन का यौगिक प्रशिक्षण है क्योंकि भागवत में कहा गया है कि इसके निरंतर सेवन से जब अशुभ वासनायें नष्ट हो जाती हैं तब भगवान श्रीकृष्ण की प्रति स्थायी प्रेम की प्राप्ति होती है, जिससे रजोगुण और तमोगुण के भाव, काम और लोभ आदि शांत हो जाते हैं और चित्त इनसे रहित होकर सत्वगुण में स्थित एवं निर्मल हो जाता है (श्रीमद्भागवत, 1/2/18-20)। जो मनुष्य इसका रसास्वादन करता है वह सांसारिक भोगों को त्यागकर ईश्वरीय आनन्द में लीन हो जाता है और उस असीम आनन्द की अनुभूति ही योग का उत्कर्ष है। इसी अवस्था प्राप्ति हेतु प्रस्तुत शोध-पत्र के अन्तर्गत योग के विविध आयामों के अध्ययन की आवश्यकता महसूस की गई।

\section{श्रीमद्भागवत में योग एवं योग के आयाम}

योग ब्रह्मा द्वारा निर्दिष्ट एक शाश्वत विज्ञान है, साधना पद्धति है। ऋषियों, तपस्वियों तथा योगियों द्वारा बतायी गई श्रेष्ठ विद्या है। यह विशेष ज्ञान जीवन के महत्त्वपूर्ण तथ्यों को दर्शाने तथा विभिन्न भौतिक व आध्यात्मिक तथ्यों को साकार करने वाला है। 'योग' शब्द की उत्पत्ति संस्कृत भाषा के 'युज' धातु से हुई है जिसका अर्थ है- जोड़ना। इस एकीकरण का अर्थ जीवात्मा तथा परमात्मा के एकीकरण से लिया जाता है। भगवान श्री कृष्ण ने गीता में योग को सर्वश्रेष्ठ सिद्ध करते हुए कहा है- तपस्वियों से योगी श्रेष्ठ, ज्ञानियों से योगी श्रेष्ठ है, कर्मयोगियों से भी योगी श्रेष्ठ है इसलिए हे अर्जुन! तू योगी बन (श्रीमद्भगवद्गीता, $6 / 46)$ ।

"जीवात्मा परमात्म संयोगो योग:" कहते हुए भगवान याज्ञवल्क्य ने जिस योग की विवेचना की है वह केवल कल्पना नहीं, अपितु दैनिक जीवन की एक अनुभूत साधना है और एक ऐसा उपाय है, जिसके द्वारा अपने साधारण मानसिक क्लेशों एवं जीवन की अन्यान्य कठिनाइयों का बहुत सुविधापूर्वक निराकरण किया जा सकता है। जीवन के विस्तृत अध्ययन के बाद ऋषियों ने यह तत्वदर्शन खोजा और प्रतिपादित किया कि व्यष्टि और 
समष्टि से संयोग हुए बिना शांति नहीं प्राप्त हो सकती। व्यक्तित्व चेतना का विराट विश्व में विस्तीर्ण एकरस चेतना में मिलने पर ही मनुष्य की अशांति का अंत हो सकता है। इस लक्ष्य के लिए निर्दिष्ट समस्त पद्धतियां योग के अंग हैं; क्योंकि योग का अर्थ ही है व्यष्टि का समष्टि से तादात्म्य, लघु का विभु, अणु का महत्, जीव का परमात्मा से पूरी तरह से जुड़ जाना, एकरस हो जाना। योग ऋषियों, तपस्वियों तथा योगियों द्वारा बनी एक ऐसी श्रेष्ठ विद्या है जो तन को सुगठित, मन को नियंत्रित और आत्मा को उद्भासित करती है। यह सभी अभ्युत्थानों का सबसे आधार एवं सारस्वत उपलब्धियों का अक्षुण्य स्त्रोत है। इसके नियमित अभ्यास से मनुष्य के सभी संशय दूर हो जाते हैं और वह अपने जीवन में परमलक्ष्य को प्राप्त कर लेता है। अपनी ऊंचाई के चरम बिंदु पर पहुंचकर योग आत्मा और परमात्मा के मिलन का अप्रतिम माध्यम बन जाता है। जैसे कि महर्षि पतंजलि ने योग को परिभाषित किया है- "योगश्चितवृत्ति निरोध:" (पातंजल योगसूत्र, 1/2)। अर्थात् समस्त चित्तवृत्तियों (मनोव्यापार) का निरोध करना ही योग है। गीता में जो कि मुख्य रूप से कर्मयोग का अभिद्यान करने वाला ग्रंथ माना गया है, कर्मों के कौशल को योग कहा गया है, "योग कर्मसु कौशलम्" (श्रीमद्भगवद्गीता, 2/59)। उपनिषदों में प्राय: जीवात्मा एवं परमात्मा के संयोग को योग कहा गया है। उपनिषदों के अनुसार जीवात्मा स्वाभाविक रुप से चैतन्य एवं सभी प्रकार की उपाधियों से अपरिछिन्न है, तथापि वह शरीर, चित्त एवं इंद्रियों से सम्बद्ध होकर भौतिक पदार्थों में अहंकार की प्रतीति से युक्त होकर कर्मों में प्रवृत्त होता है। इस प्रकार बन्ध की श्रृंखला में वह बद्ध होता जाता है। योग साधना द्वारा जीव में भौतिक पदार्थों के प्रति अहंकार की पूर्ण निवृत्ति होने पर जीवात्मा इन प्रपंचों से मुक्त होकर अपने चैतन्य स्वरूप से अवगत हो जाता है। फलस्वरूप अनादि बन्ध की श्रृंखला से मुक्त होकर परमानंदमय स्वरूप में आत्मलीन हो मुक्तिसुख का अनुभव करता है। यही योग की स्थिति है। श्रीमद् भागवत में भगवान श्री कृष्ण ही सबकी आत्मा हैं, वह योग सांख्य, सत्य, ऋत, तेज, श्री, कीर्ति और दम इन सबके परम गति परम अधिष्ठान हैं (श्रीमद्भागवत, $11 / 13 / 39$ ) । इसीलिए उद्धव कहते हैं- आप ही समस्त योगियों की गुप्त पूंजी, योगों के कारण और योगेश्वर हैं। आप ही समस्त योगों के आधार, उनके कारण और योग स्वरूप ही हैं (श्रीमद्भागवत, $11 / 7 / 14)$ । अतः श्रीमद् भागवत में योग पूर्णता की वह अवस्था है जिसमें वुत्ति कृष्णाकार हो जाती हैं। पातंजल योग सूत्र में चित्त की वृत्ति के निरोध को योग कहा गया है (पातंजल योगसूत्र, 1/2)। परन्तु भागवत के अनुसार जो साधक भगवान श्री कृष्ण का आश्रय लेकर उनके द्वारा कही गई योग साधना में संलग्न रहता है उसकी सारी कामनाएँ नष्ट हो जाती हैं और वह आत्मानन्द की अनुभूति (योग) को प्राप्त करता है (श्रीमद्भागवत, $11 / 28 / 44$ )। इस प्रकार योग के चरमावस्था में
चित्तवृत्ति शून्य नहीं अपितु कृष्णरूपी परमब्रह्म वृत्ति के साथ एकाकार हो जाती है।

वस्तुतः योग शब्द का विस्तार स्वयं व्यापक है, जिसके अंतर्गत योग की क्रियापरक पद्धतियाँ भी आती हैं, परंतु योग के उन आयामों या परंपराओं का पूर्ण विवरण तो श्रीमद्भागवत में प्राप्त नहीं होता परंतु भागवताकार ने सांकेतिक ढंग से इनकी चर्चा की है। इन्ही संकेतों के आधार पर योग के विभिन्न आयामों का उल्लेख किया गया है। जिसकी चर्चा करते हुए भगवान श्रीकृष्ण श्रीमद्भागवत में उद्धव से कहते हैं-

योगास्त्रयो मया प्रोक्ता नृणां श्रेयोविधितसमा।

ज्ञान, कर्म च भक्तिश्चनोपायोडन्योऽकुत्रचित// (श्रीमद्भागवत, 11/20/6) अर्थात मैंने ही वेदों में एवं अन्यत्र भी मनुष्यों का कल्याण करने के लिए अधिकारी भेद से तीन प्रकार के योग कर्मयोग, ज्ञानयोग और भक्तियोग का उपदेश किया है। मनुष्य के परमकल्याण के लिए योग के अतिरिक्त और कोई उपाय कही नहीं है। श्रीमद् भागवत में योग के इन्हीं रहस्यों को प्रकाशित करने हेतु प्रस्तुत शोधपत्र के अन्तर्गत योग के विविध आयामों को उजागर करने का प्रयास किया गया है।

\section{श्रीमद्भागवत में ज्ञानयोग}

प्रथम अध्याय में ज्ञानयोग का वर्णन प्राप्त होता है। ज्ञान शब्द 'ज्ञा' धातु में 'ल्युट' प्रत्यय लगाने से बनता है जिसका सामान्य अर्थ है- जानना, बोध, सच्ची जानकारी (पाणिनी, अष्टाध्यायी, $3 / 3 / 115)$ | इसका गूढ़ अर्थ होता है- आत्म साक्षात्कार, बुद्धिवृत्ति, शासत्रानुशीलन, आत्मतत्व इत्यादि। ज्ञान का व्यापक अर्थ हमारी अन्तः चेतना के उच्चतम शिखर का प्रकटीकरण है। व्यापक अर्थ में जहाँ भी चेतना का प्रकाश हो वह ज्ञान है (विद्यारण्य पंचदशी, 8/4)। तात्पर्य यह है कि व्यापक ज्ञान अनुभूति जन्य है। इस ज्ञान की शक्ति मानव को सहज ही सभी द्वन्द्वों तथा विघ्नों से पार करा देती है। गीता में भगवान श्रीकृष्ण ने भी कहा है- 'न हि ज्ञानेन सदृशं पवित्रमिह विद्यते' अर्थात् इस संसार में ज्ञान से बढ़कर पवित्र वस्तु और कोई नहीं है (श्रीमद्भगवद्गीता, 4/13)। श्रीमद्भागवत में जहाँ कहीं भी ज्ञान का प्रसंग आया है, वहाँ बड़ी युक्ति और अनुभव की भाषा में समस्त वृत्तियों से परे निर्गुण ब्रह्मतत्त्व का विवेचन हुआ है। तत्त्ववेत्ता लोग ज्ञाता और ज्ञेय के भेद से रहित अखण्ड अद्वितीय सच्चिदानन्द स्वरूप ज्ञान को ही तत्त्व कहते हैं उसी को ब्रह्म, परमात्मा और भगवान के नाम से पुकारते हैं। श्रीमद् भागवत के अनुसार विशुद्ध परमार्थ रूप, अद्वितीय तथा भीतर-बाहर के भेद से रहित परिपूर्ण ज्ञान ही सत्य वस्तु है। वह सर्वान्तवर्ती और सर्वथा निर्विकार है, उसी का नाम भगवान है (श्रीमद्भागवत, $1 / 2 / 11)$ । तात्त्विक रूप से परम सत्ता का साक्षात्कार ही यथार्थ व व्यापक ज्ञान है। श्रीमद् भागवत में इसी तात्त्विक विश्लेषण के 
आधार पर ज्ञानयोग की चर्चा की गई है। परम तत्त्व का ज्ञान प्राप्त कराने के लिए और जन्म-मृत्यु रूप संसार से मुक्ति का मार्ग बताने के लिए रूपक के माध्यम से भी उसी तत्त्व का वर्णन किया गया है। कथा-कहानियों के माध्यम से भी उसी तत्त्व का निरूपण होता है (पोद्दार, 1916, पृ० 62)। श्रीमद् भागवत में भगवान विष्णु और वासुदेव कृष्ण को ही ब्रह्म रूप में निरूपित किया गया है। श्रीकष्ण ही जगत के असंख्य जीवों की एकमात्र आत्मा है, जगत के कल्याण के लिए वे ही आत्म माया से शरीर के भांति प्रतीत होते हैं (श्रीमद्भागवत, $10 / 14 / 55$ )। ज्ञानयोग के विशेष उपदेश के रूप में भागवत के अनेक अंशों का नाम लिया जा सकता है, जैसे- गीता रूप से हंसगीता, कपिलगीता, उद्धव के प्रति भगवान के उपदेश, चतुःश्लोकी भागवत आदि। भागवत के एकादश स्कंद के 24वें अध्याय में भगवान श्रीकृष्ण विदुर को सांख्य के रूप में ज्ञानयोग का उपदेश करते हैं। श्रीमद्भागवत में ज्ञानयोग की साधन प्रणाली के अर्त्तगत भी ज्ञान, वैराग्य और भक्ति के सहित निवृत्ति मार्ग को प्रकाशित किया गया है। जो पुरुष भक्ति पूर्वक इसके श्रवण, पठन और मनन में तत्पर रहता है वह मुक्त हो जाता है।

श्रीमद्भागवत के अनुसार ब्रह्मविचार के साधन हैश्रवण, मनन, निदिध्यासन और स्वानुभूति। उनमें सहायक हैआत्मज्ञानी गुरुदेव। इनके द्वारा विचार करके देहादि अनात्म पदार्थों का निषेध कर देना चाहिए। इस प्रकार निषेध के द्वारा आत्मविषयक संदेहों को छिन्न-भिन्न करके अपने आनन्दस्वरूप में आत्मा में ही मग्न हो जाये और सब प्रकार की विषय-वासनाओं से रहित हो जाये (श्रीमद्भागवत, $11 / 28 / 23$ )। इस प्रकार श्रुतियों की भांति भागवत में भी श्रवण, मनन, निदिध्यासन और स्वानुभूति को ज्ञानयोग के साधन के रूप में स्वीकार गया है जिनका आचरण करने से अज्ञान और उसके कार्यों की निवृत्ति हो जाती है और जब संसार की समस्त आसक्तियाँ मिट जाती हैं तब भगवान के तत्व का अनुभव स्वतः ही हो जाता है। श्रीमद्भागवत में भक्ति संगत ज्ञान का वर्णन मिलता है। अतः इसमें ज्ञानयोग का जो रूप दर्शाया गया है वह सहज, सरल, ज्ञानवर्धक, उपयोगी और सर्वसुलभ है। इसे कोई भी सहज रूप में अपना सकता है।

\section{श्रीमद्भागवत में कर्मयोग}

श्रीमद्भागवत का दूसरा आयाम कर्मयोग है, जिसके अन्तर्गत कर्म को फलभोग का हेतु माना गया है। विश्व में प्रत्येक वस्तु कर्म के नियम द्वारा संचालित होती है। कर्म के बिना एक क्षण भी नहीं रहा जा सकता; क्योंकि कर्म तो मनुष्य के क्रियाकलापों का समूह है। गीता में भगवान श्रीकृष्ण कहते हैं- कोई भी पुरुष किसी काल में क्षण मात्र भी बिना कर्म किये नहीं रह सकता (श्रीमद्भगवद्गीता, 3/5)। अतः कर्म आवश्यक है परन्तु जिस भाव से जीव जैसा कर्म करता है वैसे ही शरीर से वह उस कर्म
का फल भी भोगता है (मनुस्मृति, 12/81)। इसलिए जीवन को कर्मवृत्ति से मुक्त करना मनुष्य का महत्वपूर्ण लक्ष्य है।

पुराण संदर्भकोष के अनुसार- शास्त्र विहित उत्तम क्रिया का नाम 'कर्म' है (पोद्दार, 1963, पृ. 52)। श्रीमद्भागवत में इन्द्रियों द्वारा की जाने वाली क्रियाओं को कर्म कहा जाता है। श्रीकृष्ण कहते हैं- सत्, रज और तम ये तीन गुण इन्द्रियों को उनके कर्मों में प्रेरित करते हैं और इन्द्रियाँ कर्म करती हैं (श्रीमद्भागवत, $11 / 10 / 31$ )। यद्यपि समस्त कर्ता, करण और कर्म श्रीकृष्ण की ही अभिव्यक्तियाँ हैं (श्रीमद्भागवत, $11 / 13 / 19)$ । तथापि ईश्वर अपनी माया के द्वारा प्रपंच रूप से प्रतीत होता है जिसके कारण जीवों को अविद्या से कर्तापन की भ्रांति होती है। इसलिए योगी को समस्त कर्मों को भगवान श्रीकृष्ण में ही समर्पित करना चाहिए।

जब फल की अपेक्षा से कुछ कार्य किया जाता है तो उसे कर्म कहते हैं, परन्तु जब इसके विपरीत बिना किसी फलाकांक्षा के कार्य किया जाता है तो उसे कर्मयोग कहा जाता है। श्रीमद्भागवत में भगवान श्रीकृष्ण कहते हैं कि जिनके चित्त में कर्मों और उनके फलों से वैराग्य नहीं हुआ है, उनमें दु:ख बुद्धि नहीं हुई है, वे सकाम व्यक्ति कर्मयोग के अधिकारी हैं (श्रीमद्भागवत, $11 / 20 / 7$ )। लेकिन कर्म के संदर्भ में जितने भी विधि-निषेध है, उनके अनुसार तभी तक कर्म करने चाहिए, जब तक कर्ममय जगत और उससे प्राप्त होने वाले स्वर्गादि सुखों से वैराग्य न हो जाए अथवा जब तक श्रीकृष्ण की लीला-कथा के श्रवण, कीर्तन आदि में श्रद्धा न हो जाये (श्रीमद्भागवत, $11 / 20 / 9$ ) ।

इस सृष्टि की रचना के बाद भगवान ने इस विशाल ब्रह्माण्ड के सुव्यवस्थित संचालन हेतु कुछ नियम एवं मर्यादायें (विधि-निषेध) स्थापित किये तथा कर्मानुसार फल प्राप्ति का दृढ़ सिद्धान्त बनाया ताकि यह सारा ब्रह्माण्ड एवं इसमें निवास करने वाले जीवधारी एक निश्चित विधि-विधान के अनुसार चल सके। ये व्यवस्था इतनी अकाट्य है कि भगवान भी इसका पालन करते हैं। भागवत में श्रेष्ठ कार्यों के लिए, उच्च महत्त्वाकांक्षाओं के लिए उसके समक्ष उत्तम जीव के गुणगान, उसके अनकहे लाभ और अनुग्रह की प्रशंसा का स्पष्ट विवरण मिलता है। जिसके द्वारा कर्म निवृत्ति के पथ पर बढ़ते हुए कर्मयोग को प्राप्त किया जा सकता है। इसीलिए भगवान श्रीकृष्ण ने सत्संग से कर्म निवृत्ति की बात कही है- 'सत्संग: सर्वसंगापहो हि माम्' (श्रीमद्भागवत, $11 / 12 / 2$ ) । अर्थात् जगत में जितनी आसक्तियाँ है उन्हें सत्संग नष्ट कर देता है। भगवान श्रीकृष्ण के सत्संग से अर्जुन की समस्त आसक्तियाँ समाप्त हो गयी। राजराजेश्वर पुरूरवा के मन में सत्संग के द्वारा जब विरक्ति भावोद्गार उठने लगे, तब उसने उर्वशी लोक का परित्याग कर दिया (श्रीमद्भागवत, $11 / 26 / 26)$ । ज्ञानोदय होने के कारण उसका मोह जाता रहा 
और उसने अपने हृदय में ही भगवान श्रीकृष्ण का साक्षात्कार कर लिया। अतः कर्मयोग के पथ पर अग्रसर मनुष्यों को चाहिए कि पुरूरवा की भांति सत्पुरूषों का संग करें।

\section{श्रीमद्भागवत में भक्तियोग}

योग के आयामों के क्रम में अगले आयाम में भक्तियोग का वर्णन किया गया है। श्रीमद्भागवत का आदि, मध्य और अन्त भक्तियोग परक चर्चा से ही वर्णित है। भक्ति भावों की पवित्रता है। भावनायें जीवन की जड़ हैं, मानवीय अस्तित्व का आधार है। जब ये सम्पूर्ण भावनायें भगवान को अर्पित कर दी जाती हैं तब यही भक्ति कहलाती है। भगवान के साथ सम्बन्ध जोड़ने के कारण इसे भक्तियोग कहकर योग की एक शाखा के रूप में स्वीकार किया गया है तथा ईश्वर प्राप्ति का सबसे सहज-सरल व स्वाभाविक मार्ग बताया गया है। महर्षि शाण्डिल्य ईश्वर के प्रति अपूर्व अनुराग को ही भक्ति कहते हैं (शाण्डिल्य भक्तिसूत्र, 2)। महर्षि नारद भगवान के प्रति परम प्रेम को भक्ति मानते हैं (नारद भक्तिसूत्र, 2)। महर्षि पतंजलि पातंजल योगसूत्र में ईश्वर प्रणिधान के रूप में मान्यता देते हुये कहते हैं- ईश्वर प्रणिधानाद्वा (पातंजल योगसूत्र, 1/23)। अर्थात् ईश्वर की शरण में जाकर ही जीवन का लक्ष्य प्राप्त किया जा सकता है।

भागवत में यह घोषित किया गया है कि निर्गुण ब्रह्म की उपासना देहाभिमानी जीव के लिए अत्यन्त कष्टसाध्य है, जबकि सगुण ईश्वर की उपासना सहज साध्य है। अतः श्रीकृष्ण के रूप, गुण, लीलाओं का चिंतन, उनके नामों का कीर्तन, लीलाधामों का दर्शन और अर्चाविग्रहों का पूजन ही भागवतोक्त भगवद्भक्ति के प्रधान अंग हैं और यही तत्वज्ञान का प्रधान साधन है। भागवत में इसे ही मनुष्यों का सबसे उत्तम धर्म-परम धर्म कहा गया है (श्रीमद्भागवत, $1 / 2 / 6$ )। भक्तियोग को सबसे उत्तम मार्ग बताते हुए भगवान श्रीकृष्ण उद्धव से कहते हैं कि कर्म, तपस्या, ज्ञान, वैराग्य, योग, दान, धर्म, तीर्थयात्रा, व्रत आदि अन्य साधनों के द्वारा जो प्राप्त होता है, मेरा भक्त भक्तियोग के द्वारा अनायास ही वह सब प्राप्त कर लेता है (श्रीमद्भागवत, $1 / 20 / 32$ ) । भागवत में महर्षि व्यास ने कपिल के मुख से देवाहुति के प्रति भक्ति की सारगर्भित व्याख्या करायी है। उन्होंने स्पष्ट किया है कि वेद विहित कर्म में लगे हुए जनों की भगवान के प्रति अनन्य भाव पूर्वक स्वभाविकी सात्विक प्रवृत्ति का नाम 'भक्ति' है। इसी को उन्होंने अहैतुकी भक्ति कहा है (श्रीमद्भागवत, 3/25/32)। इस अनुभव जन्य अहैतुकी भक्ति का बोध व्रज की गोपियों ने किया था। भक्ति के सभी लक्षण गोपियों के जीवन में विद्यमान है। उनका सर्वस्व श्रीकृष्ण के प्रति समर्पित है। ऐसी अहैतुकी भक्ति जिन साधनों से प्राप्त होती है वह है- गुरु की प्रेम पूर्वक सेवा, अपने को जो कुछ मिल जाये वह सब कुछ भगवान को समर्पित कर देना, भगवद्रप्रमी महात्माओं
का सत्संग, भगवान की आराधना, उनकी कथावार्ता में श्रद्धा, उनके गुण एवं लीलाओं का कीर्तन, उनके चरण कमलों का ध्यान और उनके मंदिर, मूर्ति आदि का दर्शन एवं पूजन आदि साधनों से भगवान में स्वाभाविक प्रेम हो जाता है (श्रीमद्भागवत, $7 / 7 / 30-31)$ ।

श्रीमद्भागवत में बताया गया भक्तियोग के विवेचन से दो बातें स्पष्ट होती हैं कि भक्त में समर्पण एवं प्रेम दो भागों का समावेश होना अति आवश्यक है। जिस प्रकार भागवत में भगवान श्रीकष्ण भक्ति में सम्पूर्ण समर्पण की बात कहते हैं उसी प्रकार गीता में भी भगवान श्रीकृष्ण ने अर्जुन से कहा है- 'हे अर्जुन! तू जो कर्म करता है, जो खाता है, जो हवन करता है, जो दान देता है और जो तप करता है वह सब मुझे अर्पण कर (श्रीमद्भागवत, $9 / 27 / 20)$ । महर्षि पतंजलि ने इसे ही ईश्वर प्रणिधान कहा है (पातंजल योगसूत्र, 1/23)। इस प्रकार भक्तियोग के महान प्रभाव से भक्त के सारे बन्धन कट जाते हैं और उसका हृदय भगवद्मय हो जाता है (श्रीमद्भागवत, $7 / 7 / 36$ )। तैत्तरीय उपनिषद में भी कहा गया है कि भक्तिरस को प्राप्त करके मनुष्य परमानन्दी हो जाता है (तैत्तरीय उपनिषद, 2/7)। श्रीमद्भगवद्गीता में कहा गया है कि इस रस को प्राप्त करके मनुष्य को अन्य विषयों में रस नहीं रहता (श्रीमद्भगवद्गीता, 2/59)।

\section{श्रीमद्भागवत में अष्टांगयोग}

श्रीमद्भागवत में अष्टांग योग का भी वर्णन प्राप्त होता है। योग का प्रमुख उद्देश्य कैवल्य की प्राप्ति है और इस उद्देश्य की पूर्ति के लिए विभिन्न साधनों का विवेचन किया जाता है, जिससे मनुष्य अविवेक से मुक्त होकर अपनी आत्मा को ज्ञानलोक से आलोकित करता है। इन्हीं उपायों में से एक उपाय 'अष्टांग योग' है, जिसमें शरीर और मन की समस्त क्रियाओं का निरोध कर जीवात्मा की मुक्ति ही मुख्यतः प्रतिपाद्य है।

महर्षि पतंजलि के अनुसार अष्टांगयोग'यमनियमासनप्राणायामप्रत्याहारधारणाध्यानसमाधयोडष्टावडांनि'

(पातंजल योगसूत्र, 2/29)। अर्थात् यम, नियम, आसन, प्राणायाम, प्रत्याहार, धारणा, ध्यान और समाधि ये आठ साधन योग के हैं। यम, नियम, आसन, प्राणायाम, प्रत्याहार को बहिर्रंग योग कहा जाता है और इसके विपरीत धारणा, ध्यान, समाधि को अन्तरंग योग कहा जाता है। श्रीमद्भागवत में भी भगवान कपिल अपनी माता को अष्टांग योग का मार्ग बताते हुए कहते हैं- योगस्य लक्षणं वक्ष्ये सबीजस्य नृपात्मजे। मनो येनैव विधिना प्रसन्नं याति सत्यपथम् (श्रीमद्भागवत, 3/28/1)। अर्थात् माता जी! अब मैं तुम्हें सबीज (ध्येयस्वरूप के आलम्बन से युक्त) योग का लक्षण बताता हूँ जिसके द्वारा चित्त शुद्ध एवं प्रसन्न होकर परमात्मा के मार्ग में प्रवृत्त हो जाता है। महर्षि पतंजलि भी श्रीमद्भागवत में बतायी गई इस बात का समर्थन करते हैं कि अष्टांग योग से 
चित्तशुद्धि होती है और ज्ञान का प्रकाश प्रकट हो जाता है (पातंजल योगसूत्र, 2/28)।

अष्टांग योग में प्रथम दो अंग यम और नियम पूर्णतः मनुष्य के नैतिक विकास के लिए उत्तरदायी है इनका सम्बन्ध मनुष्य के आंतरिक जीवन से है। महर्षि पतंजलि ने यम के पाँच अंग बताये हैं- अहिंसासत्यास्तेयबह्मचर्यापरिग्रिहा यमा: (पातंजल योगसूत्र, 2/30)। श्रीमद्भागवत में यम की संख्या 12 बतायी गई है- अहिंसा, सत्य, अस्तेय, असंगता, लज्जा, असंचय, आस्तिकता, ब्रह्मचर्य, मौन, स्थिरता, क्षमा और अभय (श्रीमद्भागवत, $11 / 20 / 33)$ । महर्षि पतंजलि के अनुसार नियम की संख्या पाँच हैं- शौचसंतोषतपस्वध्यायेश्वर्पणिधानानियमा: (पातंजल योगसूत्र, 2/32)। श्रीमद्भागवत में नियम की संख्या 12 बतायी गई हैशौच, जप, तप, हवन, श्रद्धा, अतिथि सेवा, पूजा, तीर्थयात्रा, परोपकार की चेष्टा, संतोष और गुरु सेवा (श्रीमद्भागवत, $11 / 20 / 34)$ ।

आसन का सामान्य अर्थ है बैठना। परन्तु योग के संदर्भ में शरीर और उसके अंगो की विशिष्ट स्थिति को आसन कहते हैं। महर्षि पतंजलि के अनुसार 'स्थिर और सुख पूर्वक बैठना ही आसन है' (पातंजल योगसूत्र, 2/46)। भागवत में भी आसन का यही भाव प्रतिपादित किया गया है'सदाडSसनजयस्थैर्य' अर्थात् उत्तम आसनों का अभ्यास करके स्थिरता पूर्वक बैठना चाहिए (श्रीमद्भागवत, $3 / 28 / 5$ )। दैवी सती ने भगवान शिव के अपमान होने पर जब शरीर छोड़ने का निश्चय किया तब वे भी आसन स्थिर करके योग मार्ग में स्थित हुए (श्रीमद्भागवत, 4/4/24-25)। जब आसन में स्थिरता का भाव आ जाता है तब किसी भी प्रकार के द्वन्द्ध विचलित नहीं कर पाते। इसीलिए महर्षि पतंजलि कहते हैं- ततोद्वन्द्वानिभिघातः (पातंजल योगसूत्र, 2/48)।

प्राण शक्ति के नियमन और विस्तृतिकरण करने का नाम प्राणायाम है। भागवत में प्राण शक्ति का उद्भव विराट पुरुष के नासाछिद्रों से माना गया है (श्रीमद्भागवत, 2/6/2)। शास्त्रोक्त विधि से अपने स्वाभाविक श्वास और प्रश्वास को रोक लेना प्राणायाम कहलाता है (पातंजल योगसूत्र, 2/49)। प्राणायाम के तीन भेद होते हैं- पूरक, रेचक, कुम्भक। श्रीमद्भागवत के अनुसार पहले आसन को जीते फिर प्राणायाम के अभ्यास के लिए कुश, मृगचर्मादि से युक्त आसन बिछाकर शरीर को स्थिर रखते हुए सुख पूर्वक बैठकर अभ्यास करें। आरम्भ में पूरक, कुम्भक और रेचक क्रम से प्राण के मार्ग का शोधन करें जिससे चित्त स्थिर और निश्चल हो जाता है (श्रीमद्भागवत, $3 / 28 / 9$ )। माता सती ने इसी विधि से योगाग्नि प्रकट कर अपने शरीर को छोड़ा था।

इन्द्रिय वृत्तियों का अपने-अपने विषयों का आहार न करके अपने-अपने केन्द्र में स्थिर हो जाना ही प्रत्याहार है। जाबालदर्शनोपनिषद् के अनुसार स्वभाव से ही विषयों में विचरण
करने वाली इन्द्रियों को बल पूर्वक विषयों से लौटा लाने को प्रत्याहार कहते हैं (जाबालदर्शनोपनिषद्, 7/1)। श्रीमद्भागवत में भी प्रत्याहार की इस प्रक्रिया में अन्य ग्रन्थों की तरह अपने चंचल मन को समेटकर भगवान श्रीक्ण्ण में लगाना है। भगवान श्रीकृष्ण का तात्पर्य है कि अंतरात्मा में स्थिर मुझ परमात्मा में ही मन को समेटकर एकाग्र करें। वे कहते हैं 'इन्द्रियों को मन के द्वारा विषयों से हटाकर अपने हृदय में ले जाना प्रत्याहार है' (श्रीमद्भागवत, $3 / 28 / 5)$ । महर्षि पतंजलि भी भागवत में बताये गये प्रत्याहार प्रक्रिया का पूर्ण समर्थन करते हैं- स्वविषयासम्प्रयोगे चित्तस्वरूपानुकार इवेन्द्रियाणां प्रत्याहारः (पातंजल योगसूत्र, $2 / 54$ )। जिस समय समस्त इन्द्रियां विषयों से हटकर श्रीहरि में निश्छल भाव से स्थित हो जाती हैं, उस समय प्रगाढ़ निद्रा में सोये हुये मनुष्य के समान जीव के रोग-द्वेशादि सारे क्लेश सर्वथा नष्ट हो जाते हैं (श्रीमद्भागवत, $3 / 7 / 13$ )। अर्थात् इन्द्रियाँ पूर्णतः वश में हो जाती हैं (पातंजल योगसूत्र, $2 / 55$ )।

बहिरंग साधनों के बाद तीन अंतरंग साधनों का आरम्भ होता है। धारणा, ध्यान, समाधि इन तीनों का सम्मिलित स्वरूप संयम कहलाता है (पातंजल योगसूत्र, 3/4)। धारणा मुख्यतः ध्यान की पूर्व तैयारी है धारणा परिपक्व होने पर ही ध्यान में प्रवेश मिलता है। 'देशबन्धश्चितस्य धारणा' (पातंजल योगसूत्र, 3/1) अर्थात् किसी स्थान विशेष में चित्त का बाधंना धारणा कहलाता है। महर्षि पतंजलि के समान भागवत में भी कहा गया है'मूलाधार आदि किसी एक देश में मन के सहित प्राणों को रिथर करना धारणा है' (श्रीमद्भागवत, $2 / 28 / 6$ )। इन्द्रियों पर विजय प्राप्त करके फिर बुद्धि के द्वारा मन को भगवान के स्थूल रूप में लगाना चाहिए। राजकुमार ध्रुव ने तपस्या करते हुए भगवान के स्थूल रूप की ही धारणा की।

धारणा की परिपक्वावस्था का नाम ही 'ध्यान' है। किसी विषय वस्तु पर एकाग्रता या चिंतन की प्रक्रिया ध्यान कहलाती है। भागवत के अनुसार निरन्तर भगवान की लीलाओं का चिंतन और चित्त को उसी में समाहित करना ध्यान है (श्रीमद्भागवत $3 / 28 / 6$ )। यहाँ भगवान श्रीकृष्ण ही एकमात्र ध्येय हैं। अतः श्रीकृष्ण की लीलाएँ समस्त वृत्तियों को अपनी ओर आकर्षित कर लेती हैं। भक्त जब उनकी लीलाओं में निमग्न हो जाता है तब किसी अन्य विषय की ओर उसकी वृत्ति आकर्षित ही नहीं हो पाती। इसीलिए ध्येय में ही अपना मनोयोग इस प्रकार करना कि केवल उसमें ही साधक निमग्न हो, किसी अन्य विषय के ओर उसकी वृत्ति आकर्षित न हो 'ध्यान' कहलाता है। महर्षि पतंजलि भी इसी बात का समर्थन करते हुए कहते हैं- तत्र प्रत्यैकतानता ध्यानम्' अर्थात् जहाँ चित्तको लगाया जाय उसीमें वृत्तिका एकतार चलना ध्यान है (पातंजल योगसूत्र, 3/2)।

समाधि योग की एक ऐसी अवस्था है जिसमें योगी चरमोत्कर्ष को प्राप्त कर मोक्ष प्राप्ति की ओर अग्रसर होता है। 
भागवत में भी ध्यान साधना की अपनी चरम स्थिति में पहुँचने पर समाधि का स्वतः लग जाना बताया गया है- समाधानं तथाडत्मनः' अर्थात् निरन्तर भगवान की लीलाओं का चिंतन और उसी में चित्त को समाधिस्थ करना समाधि है (श्रीमद्भागवत, $3 / 28 / 6)$ | ध्यान करते-करते चित्त जब ध्येयाकार में परिणत हो जाता है, उसके अपने स्वरूप का अभाव सा हो जाता है, उसकी ध्येय से भिन्न उपलब्धि नहीं होती उस समय वह ध्यान नहीं 'समाधि' कहलाता है। भगवान श्रीकृष्ण ही ध्येय हैं उनकी लीलाओं के ध्यान में प्रगाढ़ता आने पर कृष्ण स्वरूपानुकार हो जाने पर समाधि की अवस्था प्राप्त होती है। महर्षि पतंजलि भी समाधि के इसी स्वरूप का प्रतिपादन करते हैं- 'तदैवार्थमात्र निर्भासं स्वरूपशून्यमिव समाधि:' (पातंजल योगसूत्र, 3/3)। भगवान कपिल ने माता देवहूति को इसी प्रकार के समाधि योग का उपदेश दिया था जिससे माता देवहूति योग साधना के द्वारा योगाभ्यास करते हुयी समाधि में स्थित हो गयीं (श्रीमद्भागवत, $3 / 33 / 27)$ ।

यद्यपि श्रीमद्भागवत में वर्णित अष्टांग योग उपनिषदों और पतंजलिकृत योगसूत्र में प्रतिपादित अष्टांग योग का ही प्रतिनिधित्व करता है, तथापि अपनी मौलिकता एवं विशिष्टता में यह कई नये आयामों को भी प्रकट करता है। इसमें वर्णित अष्टांग योग में भक्ति का सम्पुट है। अतः समाधि साधना तभी सम्भव हो पाती है जब सम्पूर्ण समर्पण हो पाता है। जहाँ महर्षि पतंजलि समाधि अवस्था में चित्त की वृत्तियों के निरोध की बात करते हैं वहाँ महर्षि व्यास भागवत में कृष्णमय वृत्ति की अवस्था को समाधि कहते हैं। इस तरह से यह राजयोग और भक्तियोग का अनूठा समन्वय प्रस्तुत करता है।

\section{निष्कर्ष}

इस प्रकार भगवान की प्रेममयी भक्ति से जब संसार की समस्त आसक्तियाँ समाप्त हो जाती हैं, हृदय आनंद से भर जाता है, तब भगवान के तत्व का अनुभव अपने आप हो जाता है। इसीलिए उच्चभूमि में योग इसी उद्देश्य से व्यवहार में लाया जाता है और उसको निरंतर सिद्ध किया जाता है ताकि अपनी यथार्थ आत्मा का प्रत्यक्ष ज्ञान हो सके और हम इस सृष्टि के माया रूपी भव-बंधन से मुक्ति पा सकें। भागवत में वर्णित योग की विविध धारायें इसी स्वरूप का निर्धारण करती हैं। महर्षि व्यास इस बात को जानते थे कि जिस प्रकार आहार-विहार के असंयम से कायिक रोग पनपते हैं, उसी तरह विचारणा, चिंतन, मनन एवं कर्म के लिए निर्धारित नीति-मर्यादा का उल्लंघन करने के कारण मानसिक रोग उत्पन्न होते हैं। वस्तुतः शरीर व बुद्धि की तुलना में मन अधिक सूक्ष्म, विलक्षण एवं सामर्थ्यवान है। इसी कारण मनः संस्थान को व्यक्तित्व का सबसे महत्वपूर्ण घटक माना गया है। इसीलिए भागवत में भगवान श्री कृष्ण कहते हैं कि मन का

समाहित हो जाना ही योग है। जो कि एक प्रकार से मानसिक रोगों के लिए यौगिक चिकित्सा है। पूर्णयौगिक अवस्था न केवल सभी मनोविकारों से छुटकारा दिलाती है बल्कि दिव्यगुणों का अभिवर्धन व विकास भी करती है और भागवत में वर्णित योग की विविध धारायें ही मानसिक चेतना के गहन अंतराल में प्रवेश कर व्यक्तित्व की उत्कृष्टता को प्रकट करती है।

श्रीमद्भागवत में योग के विविध आयामों के सम्बन्ध में अध्ययन करने पर सबसे अधिक महत्वपूर्ण तथ्य यह उजागर होता है कि इसमें वर्णित रास पंचाध्यायी (जिसे रसों के समूह के नाम से जाना जाता है) एक 'महायोग' के रूप में प्रकट होता है। गहन विश्लेषण के आधार पर यह निष्कर्ष निकाला गया है रास जीव और शिव के एकत्व की अनुभूति है। व्यष्टि की उपाधि से परमात्मा के मिलन की कथा है। और यही परमपद एवं कैवल्य की अनुभूति है। इस प्रकार श्रीमद्भागवत में उत्त आयामों द्वारा ईश्वर से एकाकार या योगप्राप्ति की चर्चा का उल्लेख प्राप्त होता है ।

रश्मि शर्मा, पी-एच०डी०., प्रवक्ता, योग विभाग, श्री जगद्देवसिंह संस्कृत महाविद्यालय, हरिद्वार, भारत।

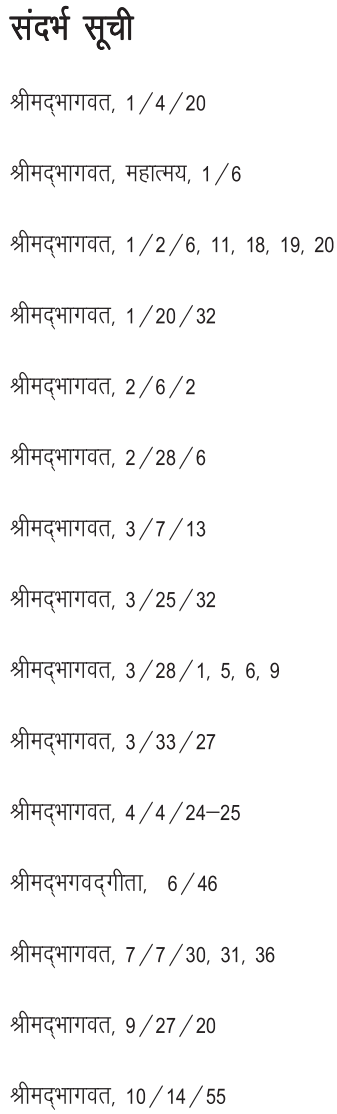


श्रीमद्भागवत, $11 / 7 / 14$

श्रीमद्भागवत, $11 / 10 / 31$

श्रीमद्भागवत, $11 / 12 / 2$

श्रीमद्भागवत, $11 / 13 / 19,39$

श्रीमद्भागवत, $11 / 20 / 6,7,9,33,34$

श्रीमद्भागवत, $11 / 26 / 26$

श्रीमद्भागवत, $11 / 28 / 23,44$

श्रीमद्भागवत, $12 / 13 / 19$

श्रीमद्भगवद्गीता, $2 / 59$

श्रीमद्भगवद्गीता, $3 / 5$

श्रीमद्भगवद्गीता, $4 / 13$

पातंजल योगसूत्र, 1/2, 23, 29

पातंजल योगसूत्र, $2 / 28,29,30,32,46,48,49,54,55$

पातंजल योगसूत्र, $3 / 1,2,3,4$
ब्रह्माण्ड प्रक्रिया- 1/170

जाबालदर्शनोपनिषद्, $7 / 1$

तैत्तरीय उपनिषद, $2 / 7$

नारद भक्तिसूत्र, 2

पाणिनी, अष्टाध्यायी, $3 / 3 / 115$

मनुस्मृति, $12 / 81$

वायुपुराण पूर्वार्द्ध, $1 / 45$

वायुपुराण, $1 / 203$

विद्यारण्य पंचदशी, $8 / 4$

शाण्डिल्य भक्तिसूत्र, 2

पोद्दार, श्रीहनुमान प्रसाद (1910). कल्याण योगांक। गीताप्रेस- गोरखपुर।

पोद्दार, श्रीहनुमान प्रसाद (1916). कल्याण-'भागवतांक / गोरखपुर- गीताप्रेस।

पोद्दार, श्रीहनुमान प्रसाद (1963). कल्याण-पुराण कथांक/ गीताप्रेस- गोरखपुर। 